Abanico Veterinario. Enero-Diciembre 2021; 11:1-15. http://dx.doi.org/10.21929/abavet2021.10 Nota Corta. Recibido: 09/09/2020. Aceptado: 02/02/2021. Publicado: 15/02/2021. Clave: 2020-78.

\title{
Estudio preliminar sobre la inhibición in vitro de nematodos gastrointestinales de ovinos con extractos acuosos de plantas forrajeras
}

Preliminary study on the in vitro inhibition of gastrointestinal nematodes from sheep with aqueous extracts of forage plants

\section{Nora Antonio-Irineo ${ }^{1} \mathrm{ID}$, Carolina Flota-Bañuelos ${ }^{2} \mathrm{ID}$, Antonio Hernández-Marín ${ }^{3} \mathrm{ID}$, Jesús Arreola-Enríquez ${ }^{1}$ ID, Silvia Fraire-Cordero ${ }^{2^{*}}$ ID}

${ }^{1}$ Colegio de Postgraduados, Campus Campeche. Carretera Haltunchén-Edzná, km. 17.5, Champotón, Campeche, México. CP. 24050. ${ }^{2}$ CONACYT-Colegio de Postgraduados, Campus Campeche. Carretera Haltunchén-Edzná, km. 17.5, Champotón, Campeche, México. CP. 24050. ${ }^{3}$ Departamento de Veterinaria y Zootecnia. Campus Irapuato-Salamanca, Universidad de Guanajuato, km 9. Carretera Irapuato-Silao, Exhacienda El Copal, Irapuato, Guanajuato. CP. 36824. *Autor de correspondencia y responsable de la investigación: Silvia Fraire-Cordero. CONACYT-Colegio de Postgraduados, Campus Campeche. Carretera Haltunchén-Edzná, km. 17.5, Champotón, Campeche, México. CP. 24050. antonio.nora@colpos.mx, cflota@colpos.mx, jahmarin@ugto.mx, jarreola@colpos.mx, frairec@colpos.mx.

\section{RESUMEN}

La alta presencia de nematodos gastrointestinales (ngi) en ovinos, es una causa de baja productividad. El objetivo fue determinar de forma preliminar la eficiencia in vitro de extractos acuosos de Gliricidia sepium, Leucaena leucocephala, Guazuma ulmifolia y Bursera simaruba a tres dosis $(0.75,1.00$ y $1.25 \mathrm{~mL})$, en la inhibición de la eclosión de huevos de ngi de ovinos. Se evaluó la eficiencia de inhibición en la eclosión de huevos $(E I H)$, identificación y proporción de géneros de larvas. El análisis de datos se realizó mediante pruebas de Chi cuadrada y análisis de varianza. Los cuatro extractos obtuvieron una ElH del $50 \%$, siendo similares $(p>0.05)$ entre ellos. La dosis de $1.25 \mathrm{~mL}$ y las combinaciones de Leucaena leucocephala y Gliricidia sepium a dosis de $1.25 \mathrm{~mL}$ obtuvieron mayor eficiencia (68.0, 85.0 y $77.0 \%$, respectivamente). Se identificaron cinco géneros de larvas (Haemonchus spp., Trichostrongylus spp., Oesophagostomum spp., Cooperia spp., y Nematodirus spp.), siendo el de mayor ( $p \leq 0.05)$ prevalencia Haemonchus spp. $(58.0 \%)$. Los cuatro extractos acuosos exhibieron actividad ovicida de ngi. No obstante, la dosis de $1.25 \mathrm{~mL}$ y los extractos de Leucaena leucocephala, Gliricidia sepium a dosis de $1.25 \mathrm{~mL}$ fueron los más eficaces.

Palabras clave: Parásitos gastrointestinales, plantas antihelmínticas, eclosión de huevos.

\begin{abstract}
The presence of gastrointestinal nematodes $(G I N)$ in sheep is a low productivity cause. This study aimed to determine the in vitro efficiency of aqueous extracts of Gliricidia sepium, Leucaena leucocephala, Guazuma ulmifolia, and Bursera simaruba at three different doses $(0.75,1.00$, and $1.25 \mathrm{~mL})$, inhibiting the egg hatching of GIN from sheep. The efficiency of inhibition in egg hatching (IEH), larval identification, and its genera proportion were evaluated. Analysis of data was performed using Chi-square tests and Analysis of variance. The four extracts obtained an IEH of $50 \%$, being similar $(p>0.05)$ between them. The $1.25 \mathrm{~mL}$ dose and its combinations of Leucaena leucocephala and Gliricidia sepium at $1.25 \mathrm{~mL}$ dose obtained the highest efficiency $(68.0,85.0$, and $77.0 \%$, respectively). Five genera of larva were identified (Haemonchus spp, Trichostrongylus spp, Oesophagostomum spp, Cooperia spp, and Nematodirus spp). The highest prevalence $(p \leq 0.05)$ was obtained by Haemonchus $\operatorname{spp}(58.0 \%)$. According to the results, the four aqueous extracts exhibited ovicidal activity. However, the $1.25 \mathrm{~mL}$ dose and the Leucaena leucocephala, Gliricidia sepium extracts at $1.25 \mathrm{~mL}$ doses were the most effective.
\end{abstract}

Keywords: Gastrointestinal parasites, anthelmintic plants, egg hatching. 


\section{INTRODUCCIÓN}

En México, el impacto económico derivado del parasitismo ocasionado por los nematodos gastrointestinales (ngi) es de 445 millones de dólares por año (Rodríguez-Vivas et al., 2017) representando un serio problema al afectar la productividad animal; traduciéndose en pérdida de apetito, peso, anemia, diarrea, retraso en el crecimiento e incluso la muerte (Rodríguez-Martínez et al., 2018). Durante décadas, se han empleado antihelmínticos químicos para el control de ngi, por su amplio espectro y facilidad de uso. Sin embargo, el uso irracional de los mismos (Closantel, Albendazol, Ivermectina y Nitroxinil), ha desarrollado resistencia en los ngi (Mondragón-Ancelmo et al., 2019) como se ha reportado para los géneros Haemonchus contortus, Teladorsagia circumcincta, Tricostrongylus spp., y Nematodirus spp., (Holsback et al., 2016).

Actualmente, se requieren alternativas novedosas y sostenibles para el control de ngi, como el uso de plantas forrajeras, las cuales aparte de ofrecer beneficios por su calidad nutricional, posean acción antihelmíntica; la cual se ha relacionado con la presencia de metabolitos secundarios, entre los que destacan: lectinas, terpenos, alcaloides, saponinas, antraquinonas, flavonoides y taninos (Oliveira et al., 2017); siendo estos últimos los que se han asociado principalmente en las funciones vitales de los nematodos (Medina et al., 2014). Se ha reportado que éstos tienen la capacidad de unirse a las proteínas estructurales (Son-de Fernex et al., 2016) y dependiendo de dónde y cómo se unen a las estructuras del nematodo, pueden inhibir la eclosión de los huevos, el desarrollo, motilidad y desenvaine larvario (Hoste et al., 2012; Son-de Fernex et al. 2016). En este sentido, plantas como Gliricidia sepium, Leucaena leucocephala, Guazuma ulmifolia y Bursera simaruba se consideran un grupo importante de plantas candidatas con potencial como alimento para animales en ambientes tropicales y subtropicales. En particular, Leucaena leucocephala y Gliricidia sepium son plantas altamente nutritivas, leguminosas y ricas en metabolitos secundarios (Son-de Fernex et al., 2012; RiveroPérez et al., 2019). Se les ha considerado por sus propiedades medicinales y antihelmínticas (Sánchez y Faria, 2013; Canul-Solís et al., 2018), aunque no se han observado de manera consistente en animales.

Por el contrario Guazuma ulmifolia y Bursera simaruba han sido poco estudiadas con el objetivo de examinar sus posibles propiedades antihelmínticas en animales, aunque en medicina tradicional son utilizadas contra enfermedades gastrointestinales y microbianas (Boligon et al., 2013); sin embargo, son altamente nutritivas y se usan ampliamente como alimento o alimento complementario para el ganado en zonas tropicales.

Por lo tanto, el objetivo de este estudio preliminar fue determinar la eficiencia in vitro de extractos acuosos de Gliricidia sepium, Leucaena leucocephala, Guazuma ulmifolia y Bursera simaruba a tres dosis $(0.75,1.00$ y $1.25 \mathrm{~mL})$, sobre la inhibición de la eclosión de huevos de nematodos gastrointestinales de ovinos. 


\section{Localización}

\section{MATERIAL Y MÉTODOS}

El estudio se llevó a cabo en el Laboratorio de Ciencia Animal del Colegio de Postgraduados, Campus Campeche, localizado en el km 17.5 carretera HaltunchénEdzná, Champotón, Campeche, México. Ubicado a 19² 29' 51.79" LN y 90³2' 45.01" LO, con una altitud de $24 \mathrm{msnm}$. El clima predominante es cálido subhúmedo con lluvias en verano, con una temperatura media anual de $26^{\circ} \mathrm{C}$ (García, 2004).

\section{Obtención del extracto acuoso}

Se seleccionaron plantas de Gliricidia sepium, Leucaena leucocephala, Guazuma ulmifolia y Bursera simaruba, con hojas verdes (jóvenes y maduras), de las cuales se recolectó $1 \mathrm{~kg}$ de cada una. Las hojas se lavaron por dos ocasiones con agua purificada para eliminar polvo y residuos; luego se cortaron en trozos de $2 \mathrm{~cm}$ y se depositaron en cubetas de plástico de $10 \mathrm{~L}$, a las cuales se les agregó $1 \mathrm{~L}$ de agua destilada, dejando en reposo por $12 \mathrm{~h}$. Después del reposo, el contenido de cada cubeta fue vertido en recipientes de aluminio de $3 \mathrm{~L}$ y colocado en parrillas de calentamiento a $80{ }^{\circ} \mathrm{C}$ durante 40 min; seguidamente, se molió con una batidora de inmersión (T-fal®) durante 5 min, para posteriormente, filtrarlo tres veces y depositarlo en recipientes de $300 \mathrm{~mL}$, previamente etiquetados por tratamiento. Finalmente se refrigeraron a $5^{\circ} \mathrm{C}$ hasta su uso (Vinueza et al., 2006).

La suspensión obtenida se consideró como solución estándar (100\%). A partir de dicha solución, se tomaron las dosis: $0.75,1.00$ y $1.25 \mathrm{~mL}$, respectivas para cada tratamiento, más un grupo testigo compuesto por agua destilada, contando con 10 repeticiones por cada combinación, como se muestra en el cuadro 1.

Cuadro 1. Distribución de tratamientos para evaluar la eficiencia de extractos acuosos de plantas forrajeras a tres dosis en la inhibición de la eclosión de nematodos gastrointestinales de ovinos

\begin{tabular}{l|ccc}
\hline Extracto acuoso & Dosis $(\mathrm{mL})$ & 0.75 & 1.00 \\
\hline Gliricidia sepium & 10 & 10 & 10 \\
Leucaena Leucocephala & 10 & 10 & 10 \\
Guazuma ulmifolia & 10 & 10 & 10 \\
Bursera simaruba & 10 & 10 & 10 \\
Agua destilada (Testigo) & 10 & 10 & 10 \\
\hline
\end{tabular}

Para cada combinación de extracto:dosis, se le determinó el contenido de fenoles totales (Folin), taninos totales (Folin + pvpp) (Makkar et al., 1993) y taninos condensados (Vainillina) (Makkar y Becker, 1993) como referencia, en la Facultad de Medicina Veterinaria y Zootecnia de la Universidad Autónoma de Yucatán, Yucatán, México (cuadro 2). 
Cuadro 2. Análisis químico de fenoles totales, taninos totales y taninos condensados de extractos acuosos de cuatro plantas forrajeras

\begin{tabular}{lcccc}
\hline Extracto acuoso & $\begin{array}{c}\text { Dosis } \\
\mathbf{m L}\end{array}$ & $\begin{array}{c}\text { Fenoles totales } \\
\mathbf{m g}\end{array}$ & $\begin{array}{c}\text { Taninos totales } \\
\mathbf{m g}\end{array}$ & $\begin{array}{c}\text { Taninos condensados } \\
\mathbf{m g}\end{array}$ \\
\hline Gliricidia sepium & 0.75 & 0.73 & 0.39 & 0.67 \\
& 1.00 & 0.97 & 0.52 & 0.89 \\
Leucaena leucocephala & 1.25 & 1.21 & 0.65 & 1.11 \\
& 0.75 & 1.99 & 0.86 & 1.17 \\
Guazuma ulmifolia & 1.00 & 2.65 & 1.1 & 1.56 \\
& 1.25 & 3.31 & 1.44 & 1.95 \\
Bursera simaruba & 0.75 & 1.00 & 0.77 & 0.19 \\
& 1.00 & 1.33 & 1.03 & 0.25 \\
& 1.25 & 1.66 & 1.29 & 0.31 \\
& 0.75 & 1.35 & 0.59 & 0.70 \\
& 1.00 & 1.8 & 0.78 & 0.93 \\
\hline
\end{tabular}

\section{Obtención de heces y carga parasitaria}

El estudio se llevó a cabo de acuerdo a los estándares de uso y cuidado de animales destinados a la investigación del Colegio de Postgraduados, México y de acuerdo a la Norma Oficial Mexicana NOM-024-ZOO-1995.

Previo al ensayo in vitro, se determinó el número de huevos por gramos de heces (h.p.g.), mediante un estudio coprológico. Las heces de ovino se obtuvieron de un rebaño perteneciente al rancho "Los Robles" ubicado en Adolfo López Mateos, Escárcega, Campeche, México, ubicado a $18^{\circ} 38^{\prime} 09.51 "$ "LN y $90^{\circ} 18^{\prime} 04.69 "$ LO, con clima cálido subhúmedo con lluvias en verano y temperatura promedio de $26{ }^{\circ} \mathrm{C}$ (García, 2004). Del cual aleatoriamente se tomaron 60 ovinos con edad promedio de 1.5 años, manejados bajo un sistema semi-estabulado con pastoreo diurno, encierro nocturno y sin desparasitación en los ocho meses anteriores a la colecta.

Todas las muestras de heces fueron homogenizadas en una sola muestra y procesadas mediante la técnica de McMaster, modificada por Rodríguez-Vivas y Cob-Galera (2005), para contabilizar el número de huevos de ngi, obteniendo un promedio de $671.6 \pm 250.4$ h.p.g., clasificándose dentro de una infestación moderada, que va de 200 a 800 h.p.g., recomendadas para establecer control parasitario (Morales et al., 2010).

\section{Identificación y proporción de géneros de larvas de ngi}

Se realizó un cultivo larvario con las heces anteriormente descritas, con duración de nueve días, siguiendo la metodología de Corticelli y Lai (1963) descrita por (Niec, 1968), con la finalidad de determinar la eficiencia de los extractos acuosos sobre la eclosión de 
huevos de ngi; donde los extractos a dosis correspondientes se aplicaron todos los días al momento de la aireación. El líquido colectado en tubos Falcon® de la fase final del cultivo larvario se centrifugó (Centrifuga, VELAB VE-4000®) a 1500 rpm (415.8 x g) durante $15 \mathrm{~min}$, para colectar las larvas por sedimentación.

Posteriormente se colocaron en refrigeración a $5^{\circ} \mathrm{C}$ por cinco horas para detener el metabolismo y ser contabilizadas mediante un microscopio estereoscópico (VELAB VE$S 3 \AA)$. Del total de larvas obtenidas de cada tratamiento se tomaron 100 , a las cuales se les agregó solución Lugol al 5\% y se observaron con un microscopio (UOP UB102i®), para ser identificadas mediante estructuras morfológicas, basadas en la extremidad anterior y/o posterior principalmente (Niec, 1968).

\section{Eficiencia en la inhibición de la eclosión de huevos}

Se determinó mediante la ecuación (1) propuesta por Álvarez et al. (2007).

$$
E I H=100 \llbracket 1-\frac{\mu T r}{\mu T} \rrbracket
$$

Dónde, ElH= Porcentaje de eficiencia en la inhibición de huevos, $\mu \mathrm{Tr}=$ Media aritmética del grupo tratado y $\mu T=$ Media aritmética del grupo testigo.

\section{Análisis estadístico}

Se utilizó un diseño completamente al azar con arreglo factorial $(5 \times 3)$ plus, donde el plus fue el tratamiento testigo. Los factores fueron: Extracto acuoso ( $G$. sepium, $L$. leucocephala, G. ulmifolia, B. simaruba y Agua destilada) y Dosis (0.75, 1.00 y $1.25 \mathrm{~mL}$ ). El análisis de los datos se realizó mediante pruebas de Chi cuadrada en las variables expresadas en conteos (\%) y análisis de varianza por el procedimiento de modelos lineales general (PROC GLM), del paquete estadístico SAS/STAT (SAS Institute Inc, 2012) en las variables numéricas. Se realizaron comparaciones de medias por la prueba de Tukey. Todos los análisis se realizaron con un nivel de significancia $\alpha=0.05$.

\section{RESULTADOS Y DISCUSIÓN}

\section{Eficiencia en la inhibición de huevos de ngi}

Se encontraron diferencias significativas $(p \leq 0.05)$ en el promedio de huevos eclosionados, siendo los tratamientos con extractos acuosos de plantas los que obtuvieron una reducción de la mitad de huevos eclosionados, comparado con el testigo. La dosis de $1.25 \mathrm{~mL}$ obtuvo una mayor $(\mathrm{p} \leq 0.05)$ reducción en la eclosión de huevos, en comparación con el testigo. Las menores $(p \leq 0.05)$ eclosiones de huevos se presentaron con los extractos de $L$. leucocephala, $G$. sepium con dosis a $1.25 \mathrm{~mL}$ y $B$. simaruba a $0.75 \mathrm{~mL}$ (figura 1). 


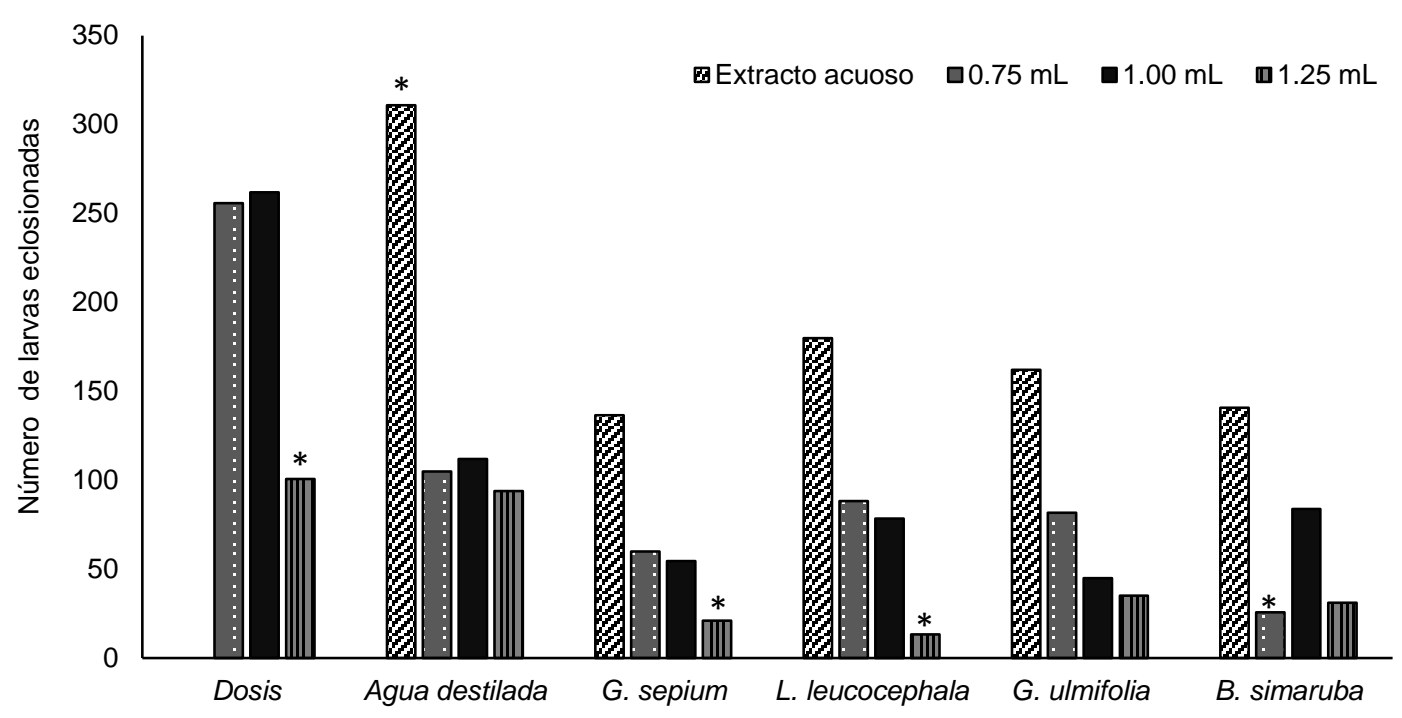

Figura 1. Promedio de larvas eclosionadas de nematodos gastrointestinales de ovinos con la adición de extractos acuosos de plantas forrajeras a tres dosis

Los resultados encontrados mostraron que los extractos acuosos afectan activamente en la eclosión de huevos de nematodos gastrointestinales. Esto sugiere que la actividad de estas plantas se asocia generalmente con la presencia de metabolitos secundarios (Torres-Acosta et al., 2016; Oliveira et al., 2017), principalmente la concentración de taninos condensados, sin descartar la participación de otros metabolitos secundarios en el efecto antihelmíntico. Se alude que el mecanismo de acción de los compuestos activos puede ser al interactuar con las membranas celulares, lo que da como resultado la desestabilización y el consiguiente aumento de la permeabilidad celular que facilita la acción sobre las proteínas intracelulares del huevo (Vieira et al., 2018) lo que inhibe su eclosión.

Sin embargo, otros mecanismos pueden contribuir al efecto observado como el reportado por Vargas-Magaña et al. (2014) y Chan-Pérez et al. (2016), quienes mencionan que los extractos vegetales posiblemente pueden inhibir la reacción de enzimas presentes en la membrana del huevo necesarias para la eclosión, o impidiendo la formación de larvas al afectar la mórula por lo que las larvas no se desarrollan completamente y no logran eclosionar. Esto resulta en una reducción en el número de larvas que nacen de los huevos; aunque, éstas son solo hipótesis que deberán ser probadas en estudios futuros.

Con relación a la EIH, los extractos acuosos fueron similares $(p>0.05)$ en la efectividad, con alrededor del $50 \%$ de inhibición. La dosis de $1.25 \mathrm{~mL}$ fue más eficiente $(p \leq 0.05)$ en comparación con el resto de las dosis evaluadas. La combinación $L$. leucocephala con dosis a $1.25 \mathrm{~mL}$ fue la más eficiente $(\mathrm{p} \leq 0.05)$, seguida de $G$. sepium a $1.25 \mathrm{~mL}$ y $B$. simaruba a $0.75 \mathrm{~mL}$. Cabe señalar que, aunque la eficiencia del extracto de G. ulmifolia 
no fue de los mejores, presentó eficiencias mayores al $59.8 \%$ con la dosis de 1.00 y 1.25 $\mathrm{mL}$ (cuadro 3).

Cuadro 3. Eficiencia de extractos acuosos de plantas forrajeras a tres dosis sobre la inhibición de huevos de nematodos gastrointestinales de ovinos

\begin{tabular}{|c|c|c|c|}
\hline Extracto acuoso & Dosis (mL) & Eficiencia (\%) & Eficiencia según extracto acuoso (\%) \\
\hline \multirow{3}{*}{ Gliricidia sepium } & 0.75 & $48.8 \mathrm{~cd}$ & \multirow{4}{*}{$56.0 \mathrm{~A}$} \\
\hline & 1.00 & $51.2 \mathrm{~d}$ & \\
\hline & 1.25 & $77.6 \mathrm{~g}$ & \\
\hline \multirow{4}{*}{ Leucaena leucocephala } & 0.75 & $16.0 \mathrm{ab}$ & \\
\hline & 1.00 & $30.0 \mathrm{bc}$ & \multirow[t]{3}{*}{$42.0 \mathrm{~A}$} \\
\hline & 1.25 & $85.7 \mathrm{~h}$ & \\
\hline & 0.75 & $22.0 \mathrm{abc}$ & \\
\hline \multirow{2}{*}{ Guazuma ulmifolia } & 1.00 & $59.8 \mathrm{de}$ & \multirow[t]{2}{*}{$48.0 \mathrm{~A}$} \\
\hline & 1.25 & 62.4 ef & \\
\hline \multirow{4}{*}{ Bursera simaruba } & 0.75 & $75.4 \mathrm{~g}$ & \multirow{4}{*}{$55.0 \mathrm{~A}$} \\
\hline & 1.00 & $25.1 \mathrm{abc}$ & \\
\hline & 1.25 & 66.7 ef & \\
\hline & 0.75 & $0.0 \mathrm{a}$ & \\
\hline \multirow{2}{*}{ Agua destilada } & 1.00 & $0.0 \mathrm{a}$ & \multirow[t]{5}{*}{$0.0 \mathrm{~B}$} \\
\hline & 1.25 & $0.0 \mathrm{a}$ & \\
\hline \multirow{3}{*}{ Eficiencia según dosis (\%) } & 0.75 & $18.0 X$ & \\
\hline & 1.00 & $16.0 x$ & \\
\hline & 1.25 & $68.0 \mathrm{Y}$ & \\
\hline
\end{tabular}

$\mathrm{a}, \mathrm{b}, \mathrm{c}, \mathrm{d}, \mathrm{e}, \mathrm{f}, \mathrm{g}, \mathrm{h}$. Letra diferente dentro de cada columna indica diferencia estadística ( $p \leq 0.05)$. A, B. Letra diferente dentro de cada columna indica diferencia significativa $(p \leq 0.05)$. $\mathrm{X}$, Y. Letra diferente dentro de cada columna indica diferencia significativa $(p \leq 0.05)$.

Todos los extractos fueron eficientes en la inhibición de eclosión de huevos de ngi, en comparación con el testigo; posiblemente por la presencia de metabolitos secundarios presentes en las hojas (Martínez-Ortíz et al., 2013; Oliveira et al., 2017) principalmente los taninos condensados a los cuales se les ha adjudicado gran parte de esta actividad; los cuales en contacto con el huevo penetran la cutícula, provocando daño ultraestructural, previniendo el desarrollo del huevo o paralizando las larvas del primer estadio (Vargas-Magaña et al., 2014). En algunas leguminosas tropicales se ha informado actividad antihelmíntica in vitro contra nematodos, sugiriendo que la actividad puede estar relacionada con la presencia de taninos (Hoste et al., 2012; GonzálezCortázar et al., 2018).

Por otra parte, plantas no leguminosas, como G. ulmifolia, su actividad puede estar relacionada con componentes fenólicos y flavonoides (Feltrin et al., 2012), los cuales han sido reportados con actividad antihelmíntica lo que puede suponer que estos tuvieron alguna influencia en la respuesta encontrada para este extracto. Sin embargo, ya que el extracto es una mezcla de compuestos bioactivos, éstos pueden estar actuando de manera individual, aditiva o sinérgica. 
Los extractos de G. sepium, L. leucocephala, en la dosis $1.25 \mathrm{~mL}$ y $B$. simaruba con dosis de $0.75 \mathrm{~mL}$ inhibieron la eclosión larvaria en un $79 \%$, esta respuesta tiene relación con el mayor contenido de taninos condensados, informado anteriormente para estas plantas (cuadro 2), lo que puede ayudar a explicar los efectos sobre la EIH con estos extractos. Estos resultados son superiores a lo reportado por Puerto et al. (2014), quienes determinaron el efecto in vitro sobre la eclosión de huevos, utilizando el extracto acuoso de G. sepium, obteniendo un $40 \%$ de inhibición y a los $50 \%$ de inhibición, con dosis de $7.90 \mathrm{mg} \mathrm{mL}^{-1}$ utilizando L. leucocephala reportado por Son-de Fernex et al. (2016), con extractos acuosos y acetónicos, más no para G. sepium, obteniendo una inhibición del $100 \%$ de los huevos a una concentración de $1.03 \mathrm{mg} \mathrm{mL}^{-1}$.

En general se observó un efecto dosis-dependiente positivo en la mayoría de los extractos de plantas; sin embargo, en el extracto a base de $B$. simaruba a menores dosis se encontraron las mayores respuestas, por lo que se propone que los metabolitos secundarios de esta planta tienen una acción más definida en esta actividad, por lo que es un buen candidato para futuras investigaciones.

Los extractos de plantas utilizados tienen una respuesta positiva hacia el control de la eclosión de los huevos de ngi por medio de los fitoquímicos presentes. Sin embargo, se requieren más estudios sobre identificación de las moléculas presentes en los extractos, eso ayudaría a comprender los mecanismos de acción involucrados en sus efectos sobre los ngi.

\section{Identificación y proporción de géneros de larvas de ngi}

Se identificaron cinco géneros de larvas de ngi, siendo Haemonchus spp., la de mayor ( $p$ $\leq$ 0.05) prevalencia con el 58.0\%, continuando Trichostrongylus spp., y Oesophagostomum spp., con 25.0 y $15.0 \%$, respectivamente. Los géneros Cooperia spp., y Nematodirus spp., consiguieron el $2.0 \%$ de predominio. En el cuadro 4 se muestran las combinaciones más efectivas en la inhibición $(p \leq 0.05)$ de huevos, según el género de ngi.

Se observa que la acción de los extractos acuosos sobre los géneros encontrados, fue positiva, ya que hay una disminución de la eclosión de huevos. Los extractos de $G$. sepium y $L$. leucocephala a $1.25 \mathrm{~mL}$ tienen mayor efecto inhibitorio sobre cuatro de los cinco géneros encontrados; en tanto que para Cooperia spp., a dosis menores de $G$. sepium y G. ulmifolia obtuvieron mayor efecto. En ese sentido, se ha reportado que para el género Cooperia spp, los compuestos fenólicos y los flavonoides han demostrado actividad (Son-de Fernex et al., 2015), lo cual sugiere que los compuestos fenólicos en estos extractos pueden tener una función importante en la inhibición de este género. 
Cuadro 4. Larvas eclosionadas según género de nematodos gastrointestinales de ovinos con la adición de extractos acuosos de plantas forrajeras a tres dosis

\begin{tabular}{|c|c|c|c|c|c|c|}
\hline \multirow{2}{*}{$\begin{array}{l}\text { Extracto } \\
\text { acuoso }\end{array}$} & \multirow{2}{*}{$\begin{array}{l}\text { Dosis } \\
(\mathrm{mL})\end{array}$} & \multicolumn{5}{|c|}{ Géneros de larvas (\%) } \\
\hline & & $\begin{array}{l}\text { Haemonchus } \\
\text { spp. }\end{array}$ & $\begin{array}{c}\text { Oesophagostomum } \\
\text { spp. }\end{array}$ & $\begin{array}{c}\text { Trichostrongylus } \\
\text { spp. }\end{array}$ & $\begin{array}{l}\text { Cooperia } \\
\text { spp. }\end{array}$ & $\begin{array}{l}\text { Nematodirus } \\
\text { spp. }\end{array}$ \\
\hline \multirow{3}{*}{ G. sepium } & 0.75 & 60.2 & 77.2 & 27.6 & $0.0^{*}$ & 0.0 \\
\hline & 1.00 & 48.7 & 38.3 & 57.7 & 50.0 & 0.0 \\
\hline & 1.25 & $29.9^{*}$ & $26.6^{*}$ & $15.7^{\star}$ & 3.8 & 0.1 \\
\hline \multirow{3}{*}{ L. leucocephala } & 0.75 & 80.9 & 60.5 & 100.0 & 100.0 & 0.0 \\
\hline & 1.00 & 96.8 & $19.1^{*}$ & 50.4 & 33.3 & 0.0 \\
\hline & 1.25 & $16.8^{*}$ & $9.4^{*}$ & $15.2^{*}$ & $3.8^{*}$ & 0.0 \\
\hline \multirow{3}{*}{ G. ulmifolia } & 0.75 & 100.0 & $13.2^{*}$ & 55.2 & 100.0 & 0.0 \\
\hline & 1.00 & 55.7 & $15.7^{*}$ & $25.2^{*}$ & $0.0^{*}$ & 0.0 \\
\hline & 1.25 & $39.6^{*}$ & $6.3^{*}$ & 44.9 & 53.8 & 0.1 \\
\hline \multirow{3}{*}{ B. simaruba } & 0.75 & $21.4^{*}$ & $28.9^{*}$ & 27.6 & 100.0 & 0.0 \\
\hline & 1.00 & 54.1 & 57.4 & 100.0 & 50.0 & 0.0 \\
\hline & 1.25 & 41.1 & 31.3 & $24.2^{*}$ & $46.2^{*}$ & 0.1 \\
\hline \multirow{3}{*}{ Agua destilada } & 0.75 & 100.0 & 100.0 & 100.0 & 100.0 & 0.0 \\
\hline & 1.00 & 100.0 & 100.0 & 100.0 & 100.0 & 0.0 \\
\hline & 1.25 & 100.0 & 100.0 & 100.0 & 100.0 & 100.0 \\
\hline
\end{tabular}

* Indica diferencia estadística ( $p \leq 0.05)$ dentro de cada columna.

Los extractos acuosos inhibieron en un $46 \%$ la eclosión de huevos de Haemonchus contortus, el cual es un helminto altamente patógeno de pequeños rumiantes, con distribución global. Las combinaciones más eficientes fueron L. leucocephala, G. sepium a $1.25 \mathrm{~mL}$ y $B$. simaruba a $0.75 \mathrm{~mL}$ con aproximadamente $77 \%$ de eficiencia, relacionándose el resultado al contenido de taninos condensados presentes. Como lo menciona Vargas-Magaña et al. (2014), quienes señalan que la actividad antihelmíntica de extractos de plantas sobre huevos de Haemonchus contortus se ha relacionado principalmente al contenido de taninos condensados y a otros metabolitos secundarios que contribuyen parcial o totalmente. Se ha especulado que incluyen alcaloides, saponinas, compuestos fenólicos (Ferreira et al., 2013) y más recientemente monoterpenoides (Goel et al., 2020).

Es importante destacar el extracto de $B$. simaruba, el cual a dosis menores obtuvieron inhibiciones mayores al $70 \%$ en Haemonchus spp., Oesophagostomum spp., y Trichostrongylus spp., por lo que es fuente de investigación.

La acción de los taninos condensados y otros compuestos vegetales secundarios se desconocen con exactitud y pueden variar con el parásito, su etapa de desarrollo y las características bioquímicas de la especie vegetal. Sin embargo, se ha identificado que la membrana externa de huevos y larvas de ngi es rica en lípidos y glicoproteínas en donde pueden unirse los taninos, lo que resulta en la acumulación de agregados (Hoste et al., 
2006) que pueden afectan el desarrollo completo de la larva, disminuyendo la eclosión del huevo o su motilidad (Martínez-Ortíz et al., 2013).

La eficacia obtenida en el presente estudio nos aporta información útil que nos permite un punto de partida para estudios enfocados a determinar dosis óptimas, identificación y aislamiento de moléculas con actividad antihelmíntica, presentes en los extractos con mayor bioactividad en el control de la eclosión de ngi.

\section{CONCLUSIONES}

Los extractos acuosos de Gliricidia sepium, Leucaena leucocephala, Guazuma ulmifolia y Bursera simaruba exhibieron actividad antihelmíntica contra huevos de ngi. No obstante, la dosis de $1.25 \mathrm{~mL}$ y los extractos de Leucaena leucocephala y Gliricidia sepium a dosis de $1.25 \mathrm{~mL}$ fueron los más eficaces. Estos resultados preliminares podrían ser una posible alternativa sustentable para la prevención y control de la parasitosis en ovinos de pelo, resaltando la necesidad de estudios sobre identificación de los compuestos bioactivos responsables de esta actividad.

\section{AGRADECIMIENTOS}

Al Consejo Nacional de Ciencia y Tecnología (CONACyT) por la beca otorgada al primer autor. Al proyecto Cátedras CONACyT 2181 "Estrategias agroecológicas para la seguridad alimentaria en zonas rurales de Campeche" del Colegio de Postgraduados campus Campeche y al propietario del Rancho "Los Robles", el Sr. Marcos Gamboa.

\section{LITERATURA CITADA}

ÁLVAREZ CV, Hernández J, Wing CR. 2007. Eficacia de aserrines para inhibir el desarrollo in vitro de larvas de parásitos gastrointestinales de ovinos. Agronomía Costarricense. 31(1):71-75. ISSN: 0377-9424. http://hdl.handle.net/10669/13815

BOLIGON AA, Feltrin AC, Athayde ML. 2013. Determination of chemical composition, antioxidant and antimicrobial properties of Guazuma ulmifolia essential oil. American Journal of Essential Oils and Natural Products. 1(1):23-27. ISSN: 2321-9114. https://www.essencejournal.com/vol1/issue1/pdf/7.1.pdf

CANUL-SOLíS J, Alvarado-Cánche C, Castillo-Sánchez L, Sandoval-Gío J, AlayónGamboa J, Peñeiro-Vázquez A, Chay-Canul A, Casanova-Lugo F, Ku-Vera J. 2018. Gliricidia sepium (Jacq.) Kunth ex Walp. Una especie arbórea multipropósito para la sustentabilidad de los agroecosistemas tropicales. Agroproductividad. 11(10):195-200. ISSN: 2594-0252. https://doi.org/10.32854/agrop.v11110.1268 
CHAN-PÉREZ JI, Torres-Acosta JFJ, Sandoval-Castro CA, Hoste H, Castañeda-Ramírez GS, Vilarem G, Mathieu C. 2016. In vitro susceptibility of ten Haemonchus contortus isolates fron different geographical origins towards acetone:wáter extracts of two tannin rich plants. Veterinary Parasitology. 217:53-60. ISSN: 0304-4017. https://doi.org/10.1016/j.vetpar.2015.11.001

FELTRIN AC, Boligon AA, Janovik V, Athayde ML. 2012. Antioxidant potential, total phenolic and flavonoid contents from the stem bark of Guazuma ulmifolia Lam. Asian Journal of Biological Sciences. 5(5):268-272. ISSN: 1996-3351. https://doi.org/10.3923/ajbs.2012.268.272

FERREIRA LE, Castro PMN, Chagas ACS, Franca SC, Beleboni RO. 2013. In vitro anthelmintic activity of aqueous lef extract of Annona muricata L. (Annonaceae) against Haemonchus contortus from sheep. Experimental Parasitology. 134:327-332. ISSN: 0014-4894. https://doi.org/10.1016/j.vetpar.2018.02.016

GARCÍA E. 2004. Modificaciones al Sistema de Clasificación Climática de Köppen (para adaptarlo a las condiciones de la República Mexicana). Quinta edición. Instituto de Geografía/UNAM. México. Pp. 90. ISBN: 970-32-1010-4. https://www.academia.edu/12911044/Modificaciones_al_sistema_de_clasificaci\%C3\%B 3n_clim\%C3\%A1tica_de_K\%C3\%B6ppen_para_adaptarlo_a_las_condiciones_de_la_R ep\%C3\%BAblica_Mexicana_2004_Enriqueta_Garc\%C3\%ADa

GOEL V, Das Singla L, Choudhury D. 2020.Cuminaldehyde induces oxidative stressmediated physical damage and death of Haemonchus contortus. Biomedicine \& Paharmacotherapy. 130:110411. ISSN: https://doi.org/10.1016/j.biopha.2020.110411

GONZÁLEZ-CORTÁZAR M, Zamilpa A, López-Arellano ME, Aguilar-Marcelino L, ReyesGuerrero DE, Olazarán-Jenkins S, Ramírez-Vargas G, Olmedo-Juárez A, Mendoza- de Gives P. 2018. Lysiloma acapulcensis leaves contain anthelmintic metabolites that reduce the gastrointestinal nematode egg population in sheep faeces. Comparative Clinical Pathology. 27:189-197. ISSN: 1618-565X. https://doi.org/10.1007/s00580-017-2577-1

HOLSBACK L, Ramsey LPA, Sanches SC, Kremer NG, Conde G, Vinícius GH, Balestrieri JV, Tomazella L. 2016. Anthelmintic efficiency of doramectin, fenbendazole, and nitroxynil, in combination or individually, in sheep worm control. Revista Brasileira de

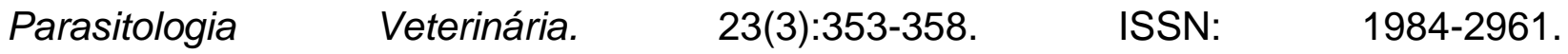
http://dx.doi.org/10.1590/S1984-29612016025. 
HOSTE H, Martínez-Ortiz-De-Montellano C, Manolaraki F, Brunet S, Ojeda-Robertos N, Fourquaux I, Torres-Acosta JFJ, Sandoval-Castro CA. 2012. Direct and indirect effects of bioactive tannin-rinch tropical and temperate legumes against nematode infections. $\begin{array}{lllll}\text { Veterinary } & \text { Parasitology. } & 186 & (1-2): 18-27 . & \text { ISSN: }\end{array}$ https://doi.org/10.1016/j.vetpar.2011.11.042

HOSTE H, Jackson F, Athanasiadou S, Thamsborg SM, Hoskin SO. 2006. The effects of tannin-rich plants on parasitic nematodes in ruminants. TRENDS in Parasitology. 22(6):253-261. ISSN: 1471-4922. https://doi.org/10.1016/j.pt.2006.04.004

MAKKAR HPS, BECKER K. 1993. Vanillin-HCl method for condensed tannins: effect of organic solvents used for extraction of tannins. Journal of Chemical Ecology. 19:613-621. ISSN: 1573-1561. http://doi.org/10.1007/BF00984996

MAKKAR HPS, Blummel M, Borowy NK, Becker K. 1993. Gravimetric determination of tannins and their correlations with chemical and protein precipitation methods. Jornal of the Science of Food and Agriculture. 61:161-165. ISSN: 1097-0010. http://doi.org/10.1002/jsfa.2740610205

MARTÍNEZ-ORTíZ de MC, Arroyo-López C, Fourquaux I, Torres-Acosta JFJ, SandovalCastro CA, Hoste H. 2013. Scanning electron microscopy of Haemonchus contortus exposed to tannin-rich plants under in vivo and in vitro conditions. Experimental $\begin{array}{lllll}\text { Parasitology. } & 133 & (3): 281- & 286 . & \text { ISSN: }\end{array}$ https://doi.org/10.1016/j.exppara.2012.11.024

MEDINA P, Guevara F, La OM, Ojeda N, Reyes E. 2014. Resistencia antihelmíntica en ovinos: una revisión de informes del sureste de México y alternativas disponibles para el control de nemátodos gastrointestinales. Pastos y Forrajes. 37 (3):257-263. ISSN: 08640394. http://scielo.sld.cu/scielo.php?script=sci_arttext\&pid=S0864-03942014000300001

MONDRAGÓN-ANCELMO J, Olmedo-Juárez A, Reyes-Guerrero DE, Ramírez-Vargas G, Ariza-Román AE, López-Arellano ME, Gives PM, Napolitano F. 2019. Detection of Gastrointestinal Nematode Populations Resistant to Albendazole and Ivermectin in Sheep. Animals. 9(10):775. ISSN: 2076-2615. http://doi.org/10.3390/ani9100775

MORALES G, Guillen TA, Pinho A, Pino L, Barrios F. 2010.Clasificación por el método Famacha y su relación con el valor de hematocrito y recuento de H.P.G. de ovinos criados en condiciones de pastoreo. Zootecnia Tropical. 28(4):545-556. ISSN: 0798-7269. http://www.scielo.org.ve/pdf/zt/v28n4/art11.pdf 
NIEC R. 1968. Cultivo e identificación de larvas infectantes de nematodos gastroentéricos del bovino y ovino. Manual Técnico 3. Instituto Nacional de Tecnología Agropecuaria (INTA). Buenos Aires, $\quad$ Argentina. $\quad$ Pp. 37. http://helminto.inta.gob.ar/Niec/Cultivo\%20e\%20Identificaci\%C3\%B3n\%20de\%20Larvas $\% 20$ Infectantes\%20de.pdf

NORMA Oficial Mexicana. NOM-024-ZOO-1995. Especificaciones y características zoosanitarias para el transporte de animales, sus productos y subproductos, productos químicos, farmacéuticos, biológicos y alimenticios para uso en animales o consumo por éstos. $\quad \mathrm{http}: / / w w w . g o b . m x / c m s / u p l o a d s / a t t a c h m e n t / f i l e / 202301 / N O M-024-Z O O-$ 1995_161095.pdf

OLIVEIRA FA, Costa JML, Lima SA, Silva RC, Ribeiro NSM, Mesquista WCJ, Rocha QC, Tangerina MPM, Villegas W. 2017. Anthelmintic activity of plant extracts from Brazilian savanna. Veterinary Parasitology. 236:121-127. ISSN: 0304-4017. https://doi.org/10.1016/j.vetpar.2017.02.005

PUERTO AM, Arece GJ, López LY, Roche Y, Molina M, Sanavria A, da Fonseca AH. 2014. Efecto in vitro de extractos acuosos de Moringa oleifera y Gliricida sepium en el desarrollo de las fases exógenas de estrongílidos gastrointestinales de ovinos. Revista de Salud Animal. 36(1):28-34. ISSN: 0253-570X. http://scielo.sld.cu/scielo.php?script=sci_arttext\&pid=S0253-570X2014000100005

RIVERO-PÉREZ N, Jaramillo-Colmenero A, Peláez-Acero A, Rivas-Jacobo $M$, Ballesteros-Rodea G, Zaragoza-Bastida A. 2019. Actividad antihelmíntica de la vaina de Leucaena leucocephala sobre nemátodos gastrointestinales de ovinos (in vitro). Abanico Veterinario. 9 (95):1-9. ISSN: 2448-6132. https://doi.org/10.21929/abavet2019.95.

RODRÍGUEZ-MARTÍNEZ R, Mendoza-de-Gives P, Aguilar-Marcelino L, López- Arellano ME, Gamboa-Angulo M, Rosas-Saito GH, Reyes-Estébanez M, García-Rubio VG. 2018. In vitro lethal activity of the nematophagous fungus Clonostachys rosea (Ascomycota: Hypocreales) against nematodes of five different taxa. BioMed Research International 2018:3501827. ISSN: 2314-6133. https://doi.org/10.1155/2018/3501827

RODRÍGUEZ-VIVAS RI, COB-GALERA, LA. 2005. Técnicas Diagnósticas en Parasitología Veterinaria. Tercera edición. 2da. Edición. Ediciones de la Universidad Autónoma de Yucatán. Mérida, México. Pp. 41-51. ISBN: 9709680458, http://bibliotecasibe.ecosur.mx/sibe/book/000049652 
RODRíGUEZ-VIVAS RI, Grisi L, Pérez LAA, Silva VH, Torres-Acosta JFJ, Sánchez FH, Romero SD, Rosario RC, Saldierna F, García CD. 2017. Potential economic impact assessment for cattle parasites in México. Review. Revista Mexicana de Ciencias Pecuarias. 8(1):61-74. ISSN: 2448-6698. http://dx.doi.org/10.22319/rmcp.v8i1.4305

SÁNCHEZ A, FARIA MJ. 2013. Efecto de la madurez de la planta en el contenido de nutrientes y la digestibilidad en una asociación Cenchrus ciliaris-Leucaena leucocephala. $\begin{array}{lllll}\text { Zootecnia } & \text { Tropical. } & 31 & \text { (1):16-23. } & \text { ISSN: }\end{array}$ http://usfx.bo/nueva/vicerrectorado/citas/AGRARIAS_7/Zootecnia/Gutierrez,\%20A.\%20J .\%20S.\%20(2014).\%20Efecto\%20de\%20la.pdf

SAS Institute. 2012. Statistical Analysis Software SAS/STAT®, versión 9.0.2, Cary, North Carolina, USA: SAS Institute Inc., ISBN: 978-1-60764-599-3. http://www.sas.com/en_us/software/analytics/stat.html\#

SON-DE FERNEX EV, Alonso DMA, Mendoza GP, Valles MB, Zamilpa A, González CM. 2016. Ovicidal activity of extracts from four plant species against the cattle nematode Cooperia punctata. Veterinaria México OA. 3(2):1-14. ISSN: 2448-6760. https://doi.org/10.21753/vmoa.3.2.365

SON-DE FERNEX EV, Alonso-Díaz MA, Mendoza-de Gives P, Valles-de la Mora B, González-Cortazar M, Zamilpa A, Castillo Gallegos E. 2015. Elucidation of Leucaena leucocephala anthelmintic-like phytochemicals and the ultrastructural damage generated to eggs of Cooperia spp. Veterinay Parasitology. 214:89-95. ISSN: 0304-4017. https://doi.org/10.1016/j.vetpar.2015.10.005

SON-DE FERNEX EV, Alonso-Díaz MA., Valles-de la Mora B, Capetillo-Leal CM. 2012. In vitro anthelmintic activity of five tropical legumes en the exsheathment and motility of Haemonchus contortus infective larvae. Experimental Parasitology. 131:413-418. ISSN: 0014-4894. https://doi.org/10.1016/j.exppara.2012.05.010

TORRES-ACOSTA JFJ, González-Pech PG, Ortiz-Ocampo GI, Rodríguez-Vivas I, TunGarrido J, Ventura-Cordero J, Castañeda-Ramírez GS, Hernández-Bolio GI, SandovalCastro CA, Chan-Pérez JI, Ortega-Pacheco A. 2016. Revalorización del bosque tropical caducifolio para la producción de rumiantes. Tropical and Subtropical Agroecosystems. 19:73-80. ISSN: 1870-0462. http://www.redalyc.org/articulo.oa?id=93945700009 
VARGAS-MAGAÑA JJ, Torres-Acosta JFJ, Aguilar-Caballero AJ, Sandoval-Castro CA, Hoste H, Chan-Pérez Jl. 2014. Anthelmintic activity of acetone-water extracts against Haemonchus contortus eggs: interactions between tannins and other plant secondary compounds. Veterinary Parasitology. 206:322-327. ISSN: 0304-4017. https://doi.org/10.1016/j.vetpar.2014.10.008

VIEIRA SAC, Oliveira SF, Goncalves LH, Dias DSG, Soares UR., Reis DÊ, Branco A, Viana CK, Mauricio DJ, Borges BM, Lima CS, Moreira BMJ. 2018. In vitro ovicidal and larvicidal activities of some saponins and flavonoids against parasitic nematodes of goats. Parasitology. 145(14):1884-1889.

ISSN: 1469-8161. http//doi.org/10.1017/S0031182018000689

VINUEZA S, Crozzoli R, Perichi G. 2006. Evaluación in vitro de extractos acuosos de plantas para el control del nemátodo agallador Meloidogyne incognita. Fitopatología de Venezuela. 19:26-31. ISSN: 0798-0035. https://www.researchgate.net/publication/48224786 